\title{
Simultaneous enumeration of protozoa and micrometazoa from marine sandy sediments
}

\author{
Slava S. Epstein* \\ Marine Science Center, Northeastern University, East Point, Nahant, Massachusetts 01908, USA
}

\begin{abstract}
The present work addresses enumeration of marine microorganisms (nanoflagellates, ciliates, and micrometazoa) from sandy sediments. The current approaches, all of which use different principles and are specific for a given group of organisms, have been modified so as to design one technique equally suitable for all the above microorganisms. This approach provides a new means to enumerate marine sediment nanoflagellates and is the first quantitative technique to enumerate ciliates from preserved samples. Perhaps most importantly, the technique also allows for simultaneous enumeration of meiobenthos so that all proto- and micrometazoan organisms can be quickly quantified from the same sample on a single preparation. The suggested technique consists of sample preservation with glutaraldehyde, extraction of target organisms by decantation, separation of the organisms from debris by isopycnic centrifugation in non-linear silica gel gradients, concentration of organisms on polycarbonate membranes, dual fluorescent staining, and enumeration under epifluorescent illumination. For marine sandy sediments, the recovery efficacy of the technique was checked by 2 independent approaches (cumulative extraction from field sediments and recovery of test organisms from azoic sediments). Both approaches showed an efficacy close or equal to $100 \%$
\end{abstract}

KEY WORDS: Sediment - Nanoflagellates - Ciliates - Meiobenthos - Epifluorescence - Enumeration

\section{INTRODUCTION}

The microscopic eukaryotes from marine sediments have long been a focus of benthic research (Fenchel 1987, Patterson et al. 1989, Giere 1993). Since the pioneering work of M. F. Mare (Mare 1942), it has become conventional to classify these organisms into meiobenthos and microbenthos. The former group comprised micrometazoa (nematodes, crustaceans, turbellarians, and other animals) whereas the latter comprised protozoa (mainly ciliated). It is only recently that the importance of other benthic protozoa, colorless and pigmented flagellates, has also been recognized (Fenchel 1975, Alongi 1986, Bak \& Nieuwland 1989, Kemp 1990, Epstein \& Shiaris 1992, Hondeveld et al. 1992). For the purpose of this study, it is convenient to place the smallest ( 2 to $20 \mu \mathrm{m}$ in length) flagellates into a separate group. Following the existing benthic terminology

•E-mail: sepstein@lynx.dac.neu.edu
(Mare 1942) as well as by analogy to planktonic research (Sieburth et al. 1978), this group will be called nanobenthos.

The importance of nano-, micro-, and meiobenthos in the structure and function of benthic communities is no longer questioned. Enumeration of these organisms has become an indispensable part of ecological studies of sediment communities. For quantification of nano-, micro-, and meiobenthos, a number of different approaches have been advanced, tested, and used.

Nanobenthos can be best enumerated by using epifluorescence microscopy, as this group always includes a large number of small (2 to $5 \mu \mathrm{m}$ ) cells difficult to observe using conventional light microscopy, especially in detritus rich environments such as sediments or turbid waters (Alongi 1990, Lovejoy et al. 1993 , Starink et al. 1994). The use of fluorochromes implies the need for thorough separation of nanobenthos from sediment/detrital particles which would otherwise make counts unreliable due to background fluorescence and masking effect. Thus, nanobenthic organ- 
isms have to be preserved, separated from non-living material, stained, and concentrated on polycarbonate filters. For fine freshwater sediments, such procedures have been developed by Starink et al. (1994). This technique works remarkably well in freshwater muddy and gyttja sediments and successfully solves the problem of high detection limit of other approaches (Alongi 1986, Gasol 1993). However, in unmodified form, it may not be applicable to sandy sediments from which microorganisms have to be first extracted. This limits the number of approaches available for enumeration of nanobenthos in sandy sediments to only one technique (Bak \& Nieuwland 1989). This approach has been gaining acceptance (cf. Bak et al. 1991, Bak \& Nieuwland 1993. Hondeveld et al. 1994) but, on several occasions (Starink et al. 1994, Starink pers. comm., present work), was reported to produce unreliable counts due to high background fluorescence.

Microbenthos so far has been enumerated mainly by live counting. The widely used sea-ice extraction technique (Uhlig 1964) has been largely replaced by the more accurate silica gel approach (Alongi 1986, 1993). The latter employs a dense Percoll-sorbitol mixture to separate various organisms, including microbenthic, from sediment particles. This approach prohibits fixation by aldehydes and some other fixatives as well as the use of several fluorescent dyes because of their gelling effects on silica gels (Alongi 1993, Gasol 1993). The technique in its present form makes it imperative to examine samples immediately upon collection and is incompatible with enumeration protocols for nanoflagellates (Bak \& Nieuwland 1989, Starink et al. 1994). This is in sharp contrast to planktonic research where ciliates and flagellates are conveniently (and simultaneously) enumerated from preserved samples by epifluorescence microscopy (Caron 1983, Sherr et al. 1993).

Enumeration of meiofauna is conventionally done by sample shaking/decantation/elutriation either with or without dense substances added to help meiobenthos flotation (for review of techniques, see McIntyre \& Warwick 1984, Giere 1993). Simple and effective for enumeration of target organisms, these techniques in their present form do not allow for simultaneous enumeration of either nano- or microbenthos.

From the quick overview of methods available for direct counting of nano-, micro-, and meiobenthos, several conclusions can be made. (1) For nanobenthos enumeration, a reliable approach is restricted to freshwater fine sediments (Starink et al. 1994). Its marine applications either have not been tested or require modifications. (2) Quantification of microbenthos presently relies on live counts (Alongi 1986), which precludes the use of common fixatives and fluorochromes. Thus, microbenthos can not be quantified simultaneously with nanobenthos. (3) Meiobenthos can be enumerated by several well-tested approaches (McIntyre \& Warwick 1984, Giere 1993). The relevant techniques are sufficiently different from those for nano- and microbenthos and represent a third set of protocols to be run in parallel if all the eukaryotic microorganisms are to be enumerated. As a result, community-level studies of marine benthos, by definition involving a variety of different organisms and large numbers of samples, are limited by inability to process efficiently the required number of samples. The purpose of this study was to identify a quick, efficient, and reliable enumeration protocol suitable for simultaneous enumeration of all the above organisms from a single preserved sediment sample. This was achieved for marine sandy sediments. The protocol is based on sample preservation with glutaraldehyde, extraction of microorganisms by repeated sample washing via shaking/collection of supernatants, separation of extracted organisms from debris by isopycnic centrifugation in Percoll density gradient (Starink et al 1994), and dual fluorescent staining of organisms concentrated on polycarbonate membranes (Sherr et al 1993). The protocol has efficiency close to $100 \%$ and can reduce several-fold the amount of time otherwise needed to process 3 separate sets of samples for nanomicro-, and meiobenthic organisms.

\section{MATERIAL AND METHODS}

Study area. Sand samples were collected near the low tidal line of a quiet tidal flat in Massachusetts Bay. near the Marine Science Center of Northeastern University, Nahant, Massachusetts, USA, about $10 \mathrm{~km}$ northeast of Boston. The sand was well sorted with median grain size around $180 \mu \mathrm{m}$. Samples were taken by coring the tidal flat sediments to a depth of $2 \mathrm{~cm}$ with a plastic tube $(5.5 \mathrm{~mm}$ i.d.) equipped with a plunger. All cores were $0.48 \pm 0.05$ (SE) $\mathrm{cm}^{3}$ Randomly taken cores were combined by threes. The combined samples $\left(1.44 \pm 0.10 \mathrm{~cm}^{3}\right)$, hereafter referred to simply as samples, were used as individual replicates in all the experiments. Except for those used for live counting, these samples were preserved with glutaraldehyde ( 1 to $1.5 \%$ final concentration) added to various Percoll solutions (see below), were stored refrigerated, and processed within a few days upon collection.

Extraction of nano-, micro-, and meiobenthic organisms. Preserved samples: Extraction of preserved nano-, micro-, and meiobenthos was done by sample shaking/collection of supernatants. The main varied parameters were the density and the amount of the washing solution. The different densities were created by adding varying amounts of silica gel Percoll 
(Sigma, St. Louis, MO, USA) to filter sterilized $(0.2 \mu \mathrm{m}$ pore-size membranes) natural sea water, hereafter called particle free sea water (PFSW). The following Percoll dilutions in PFSW were employed: $0,25,50$, and $75 \%$. To keep washing solutions isoosmotic to sea water, PFSW used to dilute Percoll was appropriately concentrated by slow evaporation. To all the solutions, glutaraldehyde was added to $1.5 \%$ final concentration prior to extraction; these solutions will be called Gl-PFSW. Two groups of experiments were conducted to test these solutions as washing agents in the shaking/decantation procedure.

Experiments with field sediments. Two triplicate experiments were performed. In Expt 1, samples were placed into $12 \times 75 \mathrm{~mm}$ disposable plastic tubes and preserved with $4 \mathrm{ml}$ of one of the 4 washing solutions to be tested. Samples were gently resuspended, shaken by hand for $10 \mathrm{~s}$, and, within a few seconds, the first supernatant was pipetted off. Washing procedure followed by supernatant collection was repeated up to 15 times per sample; each time $4 \mathrm{ml}$ of fresh washing solution was added.

In Expt 2, the variables were the amount of washing solution per sample and the geometry of the vial where washing was carried out. The samples were placed into $25 \times 150 \mathrm{~mm}$ culture vials; the amount of washing solution was $6 \mathrm{ml}$. For comparative purposes, control samples were processed as in Expt 1. Only Gl-PFSW was employed to extract the organisms; the number of washes was 5 to 10 .

Recovery experiments from azoic sediments. The absolute efficiency of the extraction protocol from Expt 1 was checked by its ability to recover organisms added in known number to azoic sediments.

Azoic sediments were obtained by drying field sediments at $80^{\circ} \mathrm{C}$ for $12 \mathrm{~h}$ followed by overnight freezing at $-20^{\circ} \mathrm{C}$. Cultures of Rhynchomonas nasuta ATCC 50105, heterotrophic flagellates 7 to $12 \mu \mathrm{m}$ in length, were used as model nanobenthic organisms. Cultures of a scuticociliate Cyclidium sp., 20 to $30 \mu \mathrm{m}$ in length, previously isolated from marine sediments, represented microbenthos. Mixed assemblages of nematodes exemplified meiobenthos; these were hand collected upon shaking/decantation of non-preserved field samples of intertidal sand. The test organisms, all suspended in $1 \mathrm{ml}$ of PFSW, were placed in $12 \times 75 \mathrm{~mm}$ plastic disposable vials to which $1.44 \mathrm{~cm}^{3}$ of azoic sediment was added from the top. Per replicate, $4 \times 10^{4}$, $6 \times 10^{2}$, and 50 individuals of, respectively, $R$. nasuta, Cyclidium sp., and nematodes were added. After 10 to $15 \mathrm{~min}$, the organisms were recovered by sample shaking/supernatant collection as described above for Expt 1. Aliquots of Gl-PFSW and $75 \%$ Percoll in GlPFSW, $4 \mathrm{ml}$ at a time, were used as alternative washing solutions; the first addition of washing solution acted simultaneously as a fixation step. Total number of washes was 5 . All experiments were conducted in triplicate. Blanks were run to check if azoic sediments contained objects which could be confused with added proto- or metazoa.

Live counts: Live counts of ciliates in triplicate samples were performed under a dissecting microscope immediately after ciliate extraction by the sea-ice technique (Uhlig 1964) for $2.5 \mathrm{~h}$.

Separation of extracted organisms from detrital particles. Direct counting of nano- and microbenthic organisms washed from the samples and contained in the supernatants proved to be unreliable because of an overwhelming number of suspended sediment/detrital particles causing unacceptably high background fluorescence.

Separation of these particles from the extracted organisms was based on the differences in their specific densities. The separation was achieved by an isopycnic centrifugation in non-linear Percoll gradients. The gradients, hereafter called Percoll gradients, were prepared after Starink et al. (1994) with minor modifications. In short, $4.5 \mathrm{ml}$ of $50 \%$ Percoll solution in twice concentrated PFSW were placed into $7 \mathrm{ml}$ polycarbonate tubes and centrifuged (RC-2, Sorvall Instruments, Wilmington, DE, USA) in an SS 34 angle rotor at $30000 \times g$ for $15 \mathrm{~min}$. This protocol was chosen after extensive tests on the effect of centrifugation time on the shape of the gradient profile. For the latter to be reconstructed, precise $50 \mu$ subsamples were taken along the vertical gradient at $5 \mathrm{~mm}$ intervals and weighed on analytical balances (Torsion Balance Co., Clifton, NJ, USA, model EA-1, $0.1 \mu$ g readability).

Two-ml subsamples of extracted organisms (see above) were carefully loaded on the top of the gradients and centrifuged in a HS-282 swing-out rotor at $5000 \times g$ for $15 \mathrm{~min}$ (minor modification from Starink et al. 1994). This resulted in pelletizing detritus particles; supernatants with floating organisms were decanted and stained (see below).

To determine the position of nano-, micro-, and meiobenthic organisms in the Percoll gradients, the supernatants in some treatments were not decanted but were divided into vertical layers. This was done by serial pipetting of $f$ the upper $m$ of the gradient in $a$ step-wise manner. Each supernatant was thus divided into 6 fractions representing 6 vertical layers. The pellets at the bottom were checked for the presence of organisms by direct light microscopy.

Staining and enumeration. Several different staining protocols were qualitatively evaluated. DAPI $\left(4^{\prime}, 6-\right.$ diamidino-2-phenylindol; this and other stains from Sigma) was used at 1.0, 5.0 (Porter \& Feig 1980, Schallenberg et al. 1989), and $10.0 \mu \mathrm{g} \mathrm{m}^{-1}$ final concentration. Fluorescein isothiocyanate (FITC) was used after 
Sherr et al. (1993) at 100 and $400 \mu \mathrm{g} \mathrm{ml}^{-1}$ final concentrations. Primulin was used after Caron (1983) at $250 \mu \mathrm{g} \mathrm{ml}^{-1}$ final concentration. A DAPI/FITC dual staining procedure was performed as described in Sherr \& Sherr (1983) and Sherr et al. (1993) at 4 and $400 \mu \mathrm{g} \mathrm{ml}^{-1}$ as well as in a slightly modified form at 10 and $100 \mu \mathrm{g} \mathrm{ml}^{-1}$ respective dye final concentrations.

Stained organisms were concentrated on Poretics 2.0 um pore-size black polycarbonate membranes (Fisher Scientific, Pittsburg, PA, USA), with vacuum never exceeding $40 \mathrm{~mm} \mathrm{Hg}$, and washed for $5 \mathrm{~min}$ with Gl-PFSW. Slides were examined under Leitz Dialux 20 or Zeiss Axiophot microscopes equipped for epifluorescence [ $\mathrm{nm}$ ranges of used excitation filters/beam splitters/barrier filters were, respectively, as follows: Leitz filter blocks 513410 (DAPI, primulin; $340380 /$ 400/430) and 513417 (FITC; $390-490 / 510 / 515) ;$ Zeiss filter blocks 9108460094 (DAPI; 365/400/450) and 9108460019 (FITC, autofluorescence, glutaraldehyde induced fluorescence, $485 / 505 / 530$ )l. To enumerate meiobenthos, entire filters were scanned at 40 to $100 x$; to enumerate microbenthos, halves of the filters were scanned at 100 to $200 \times_{i}$ to enumerate nanobenthos, a set number of microscopic fields (not less than 15) were examined at 400 to $1000 \times$. Flagellates were classified into nano- or microbenthos on the basis of size with $20 \mu \mathrm{m}$ cut off length; only colorless forms were counted. Ciliates were identified to generic, family, or in some instances order level; meiobenthos was counted at the phylum (nematodes) or suborder (harpacticoid copepods) level.

Statistics. Statistical analyses were performed after Sokal \& Rohlf (1981). The Kruskal-Wallis non-parametric test was used throughout the study.

\section{RESULTS}

\section{Staining and counting}

Counting organisms on the surface of polycarbonate membranes did not pose any problem provided (1) there was no interference from detritus, and (2) appropriate fluorescent staining of target organisms was achieved. Meiobenthic organisms were an exception in that they did not require any staining; their natural autofluorescence, enhanced by fixative induced fluorescence, particularly strong because of the use of glutaraldehyde (Sherr et al. 1993), was bright greenish yellow.

Dual DAPI/FITC fluorochrome labeling, first suggested by Sherr \& Sherr (1983; an excellent description of a variety of relevant protocols can be found in Sherr et al. 1993), worked the best for the purposes of this study. It was found necessary to increase the final
DAPI concentration to $1.0 \mu \mathrm{g} \mathrm{m}^{-1}$ and to decrease final FITC concentration to $100 \mathrm{\mu g} \mathrm{ml}^{-1}$ (higher FITC concentrations lead to overstaining of microbenthos). In this case, the FITC staining resolved details of general morphology and cilia/flagella placement whereas DAPI staining visualized well nuclei of most of the protozoa. The combination of both provided an opportunity for some taxonomic identification of nano- and microbenthos otherwise rarely possible under epifluorescent illumination. Meiobenthic animals were also stained and could be easily counted though the morphological details of taxonomic importance were difficult to observe.

No individual dye provided adequate staining for all the organisms in question. DAPI, even when used at high concentrations ( $\left.>10 \mu \mathrm{g} \mathrm{ml}^{-1}\right)$ as suggested earlier (Sherr et al. 1993), did not satisfactorily stain nuclei of many benthic ciliates (Tracheloraphis, Geleia, Kentrophoros, other karyorelicteans) as well as of a number of nano- and microflagellates. Under such conditions, a varying number of the specimens might be easily missed if stained solely with DAPI. The problem could be corrected by using dyes of more general action such as FITC (Sherr \& Sherr 1983) and primulin (Caron 1983). These more general dyes stained well most of the protozoa. However, certain organisms, especially ciliates from the order Hypotrichida, were unrecognizable as such and looked similar to shapeless pieces of detritus. These would have been missed during enumeration had they not been stained with DAPI, which revealed typical macro- and micronuclei inside otherwise shapeless objects. Thus, the limitations of the single dyes did not overlap, and the dual DAPI/FITC staining was largely free from the drawbacks of the individual dyes.

\section{Tests of Percoll gradients}

The major purpose of using gradiented Percoll solutions was to separate living and non-living material upon extraction from the sample (see below). This could be done on the basis of specific density differences between live organisms and detritus (Starink et al. 1994). The ideal density profile would keep all the target organisms afloat while letting the detrital particles settle. From earlier works (Jonge \& Bouwman 1974, Schwinghamer 1981, Alongi 1986), a pilot study (data not shown), and present experiments (see Fig. 2), it was known that an overwhelming majority of the nanoflagellates, ciliates, nematodes, copepods, turbellarians, and even hard-bodied organisms such as foraminiferans and ostracods had specific density below $1.15 \mathrm{~g} \mathrm{ml}^{-1}$. Therefore, gradiented solutions were supposed to contain a layer with specific density 


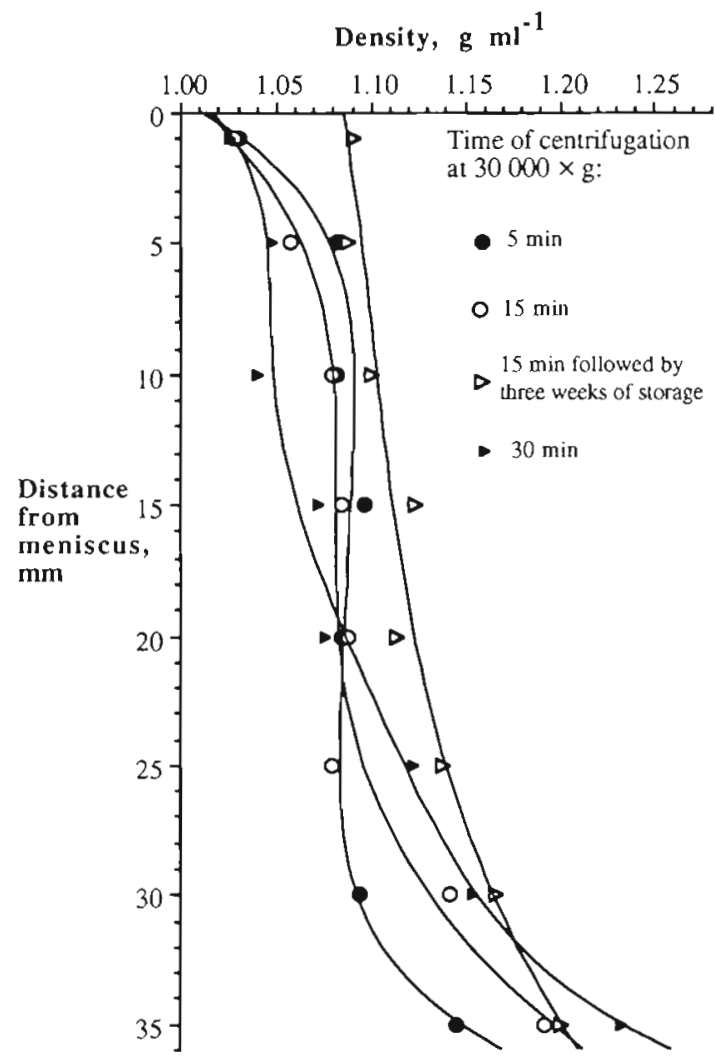

Fig. 2. Effect of centrifugation time and storage on the shape of a self-formed non-linear density gradient of $50 \%$ Percoll solution in sea water

$\geq 1.15 \mathrm{~g} \mathrm{ml}^{-1}$; this layer would act as a barrier through which the target organisms would not be able to pass. This layer developed well at centrifugation time $\geq 15$ min at $30000 \times g$ (Fig. 1). The performance of gradients was stable over time (Fig. 1).

Samples were loaded on the top of Percoll gradients and separated from detritus by the second centrifugation. The actual position of target organisms in the gradients is shown in Fig. 2. Upper, diluted fractions never contained any organism. The deeper, denser layers of Percoll retained the organisms but not detritus. From the shape of the profile it was clear that organisms suspended in gradients represented most, if not all, of those originally loaded on the Percoll gradient. This was confirmed by examination of pelletized detritus which was almost completely devoid of organisms other than diatoms

It was concluded that the mixture of large quantities of detrital material with preserved nano-, micro-, and meiobenthic organisms could be effectively separated by centrifugation in non-linear Percoll gradients. There was no gelling effect of either fixative (1 to $1.5 \%$ glutaraldehyde) or fluorochromes (FITC, DAPI) on Percoll gradients.

\section{Extraction of nano-, micro-, and meiobenthic organisms}

Preserved samples

Experiments with field sediments. The results of Expt 1 showed a gradual increase in the cumulative number of organisms extracted from the sample by its washing (Fig. $3 \mathrm{~A}-\mathrm{C}$ ). The first 5 washes brought 72 , 92 , and $88 \%$ of extractable (obtained by 15 washes) nano-, micro-, and meiobenthos. The following 5 washes increased the cumulative recovery to, respectively, 91, 98, and 96\%. Further washing (closed circles in Fig. 3) extracted relatively few additional organisms. This tendency was similar in all the treatments irrespective of the specific density of the washing solution. The total number of extractable organisms was statistically indistinguishable $(0.01 \leq \alpha \leq 0.05)$ among treatments.

The amount of washing fluid as well as geometry of the vial in which extraction took place influenced the outcome of extraction. In Expt 1 and control trials of

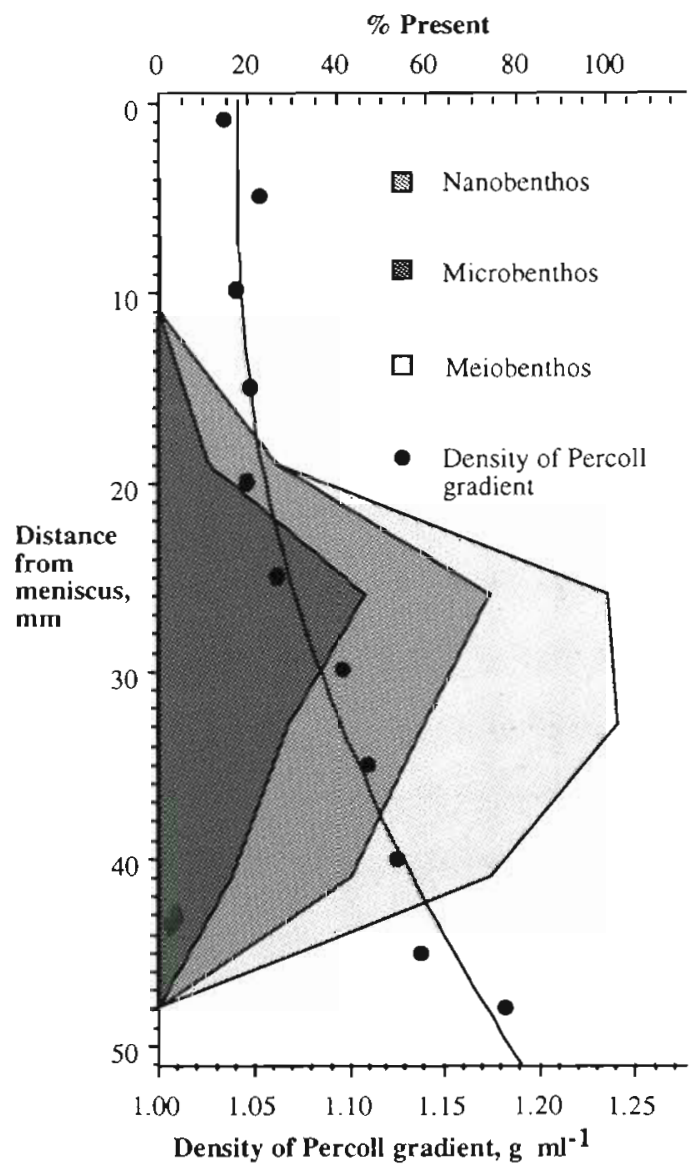

Fig. 2. Vertical distribution of nano-, micro-, and meiobenthos along the Percoll density gradient 


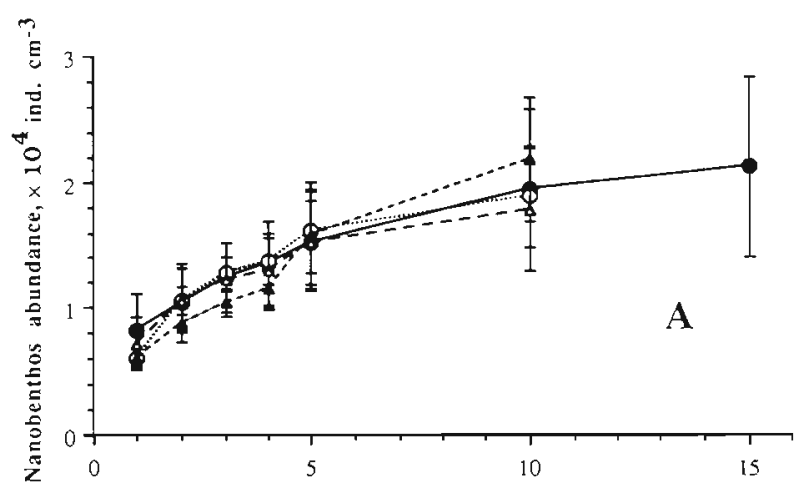

Recovery experiments from azoic sediments. The recovery experiments were designed to directly test the absolute efficacy of selected extraction protocols. When Gl-PFSW was used for washing solutions to extract added organisms, $113 \pm 13$ (SE) \% of nanobenthos, $91 \pm 39 \%$ of microbenthos, and $100 \pm 29 \%$ of meiobenthos were recovered (Fig. 5). There was no statistically significant difference $(\alpha=0.01)$ between the numbers of added versus recovered organisms of either group. Recovery by $75 \%$ Percoll in PFSW was also reasonably close to $100 \%$ (data not shown).

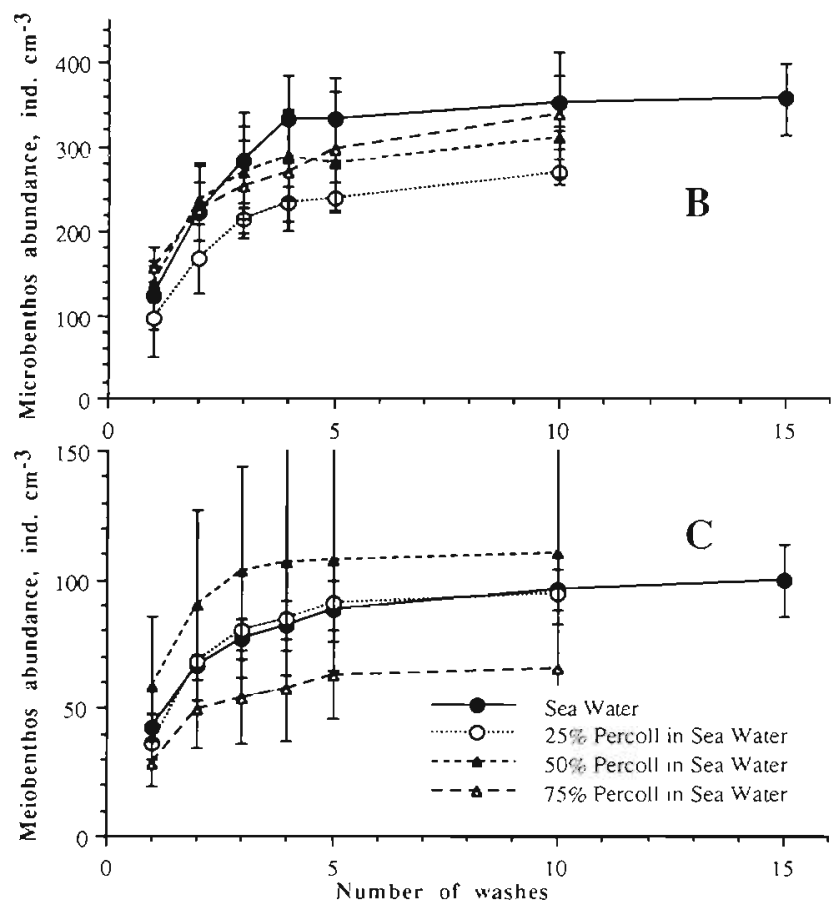

Fig. 3. Cumulative extraction of microorganisms in sediment by sample decantation with varied density of washing fluid. (A) Nanobenthos, (B) microbenthos (ciliates only), (C) meiobenthos (97\% represented by nematodes)

Expt 2 (open circles in Fig. 4) the same small vials and equally low amounts of washing fluid per decantation cycle $12.8: 1 \mathrm{w} / \mathrm{w}$ washing-fluid-to-sample (WF/S) ratiol were used. Consequently, the rates of extraction were similar. Once these variables increased (closed circles in Fig. 4; 4.2:1 WF/S ratio), the same number of organisms could be extracted twice as fast. As Fig. 4 shows, 5 washes in Expt 2 (closed circles) brought statistically the same amount of microorganisms as 10 washes by the less efficient protocol (open circles). This translates, assuming that 15 washes (Fig. 3) recovered $100 \%$ of extractable microorganisms, into $\geq 90 \%$ (nanobenthos) and $\geq 95 \%$ (micro- and meiobenthos) recovery by 5 washes in Expt 2
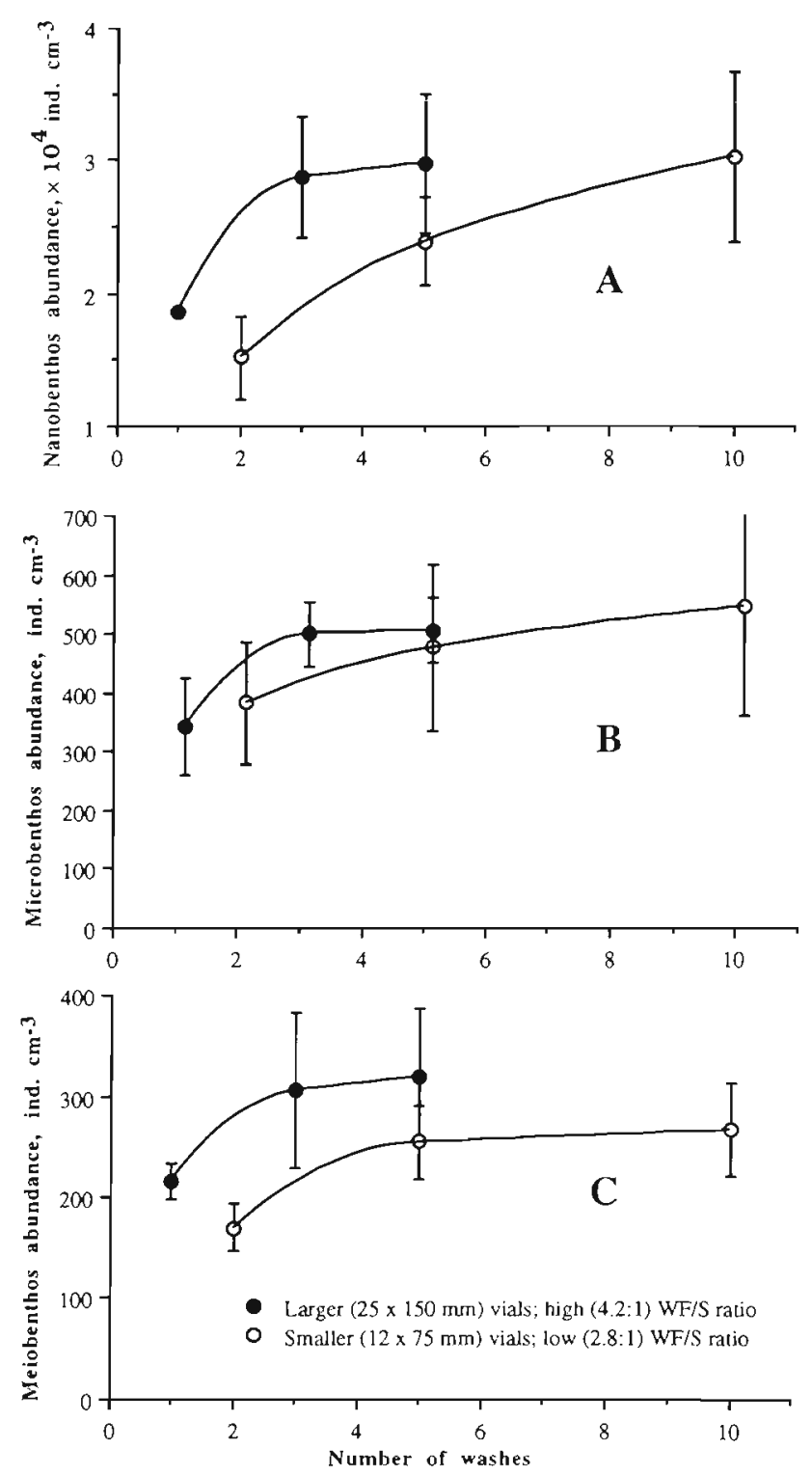

Fig. 4. Cumulative extraction of microorganisms in sediment by sample decantation with varied vial size and amount of washing fluid. (A) Nanobenthos, (B) microbenthos (ciliates only), (C) meiobenthos ( $98 \%$ represented by nematodes) 


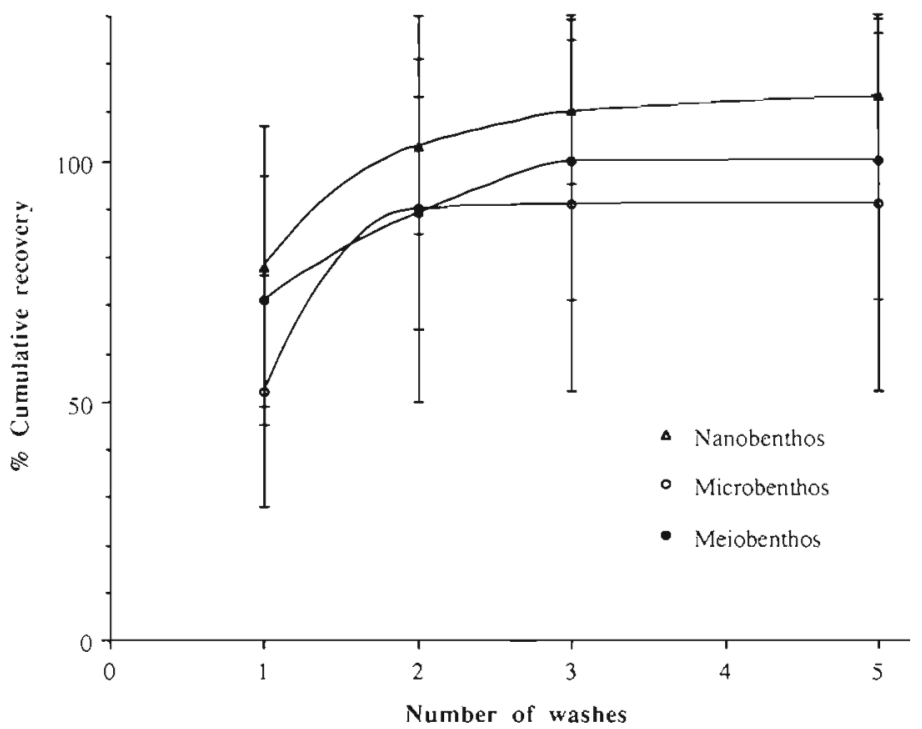

Fig. 5. Percent recovery of test microorganisms added in known numbers to azoic sediments

Live counts

Live counts were performed only for benthic ciliates The total ciliate density from live counting was $180 \pm$ 39 (SE) ind $\mathrm{cm}^{-3}$ which was significantly $(\alpha=0.01)$ lower than the total ciliate density obtained in Expt 1 (356 \pm 42 ind. $\mathrm{cm}^{-3}$; Fig. 3). The degree of undercounting was similar in all ciliate taxa except large karyorelicteans. The latter were enumerated by live counting and from preserved samples with similar efficiency (data not shown).

\section{DISCUSSION}

It was argued in the 'Introduction' that, at present, there is a lack of techniques to enumerate marine benthic microorganisms. The existing approaches are specific for either nano-, micro-, or meiobenthos so that no 2 groups can be enumerated at the same time and from the same sample. Considering the importance of nano-, micro-, and meiobenthos in the energetics of marine sediment communities and the importance of ecological studies of their interactions, it seemed desirable to identify a single approach allowing for simultaneous and reliable enumeration of all the above organisms from a single sample. Earlier attempts (Epstein \& Shiaris 1992, Berninger \& Epstein 1995) resulted in rather time-consuming protocols. The goal of the present study was to identify an effort-saving approach, check its absolute efficiency, and work out the details of its application to marine sandy sediments.
The basic starting point of this project was that the desired enumeration protocol was to be based on epifluorescence microscopy as the other approaches might not provide for reliable quantification of nanobenthos. This required all the microorganisms in question to be (1) quantitatively extracted from preserved samples, and (2) purified to eliminate detritus particles. For extraction, a sample shaking/decantation of a type used to enumerate meiobenthos (McIntyre \& Warwick 1984, Giere 1993) was chosen for extensive testing. Extraction by silica gel (Jonge \& Bouwman 1974, Nichols 1979, Schwinghamer 1981, Alongi 1986) could not be used because it would prohibit the use of common fixatives and fluorochromes. In an attempt to further purify the extracted organisms and clean them from detritus, an isopycnic centrifugation in density gradients (Starink et al. 1994) was tested. The entire protocol from the latter paper could not be used as it set too high a detection limit for any organism less abundant than nanobenthos.

In Expt 1, extraction of benthic microorganisms by sample washing/collection of supernatants was tested to find the optimal density of washing fluid. The specific density was varied by adding silica gel (Percoll) to appropriately concentrated sea water. However, the use of Percoll as a heavy agent proved to be unnecessary. Provided the supernatant was collected within a few seconds upon sample resuspension, natural sea water proved to be as good a washing fluid as heavier Percoll solutions (Fig 3).

In Expt 1, the extraction conditions were deliberately made suboptimal. The $W F / S$ ratio was made low (under $3: 1$ ) to decrease the extraction efficacy of individual washes (Schwinghamer 1981, Starink et al. 1994). In addition, vials in which extraction took place were chosen with a rather small diameter (12 $\mathrm{mm}$ o.d.) to increase trapping of organisms by sinking sediment particles. The idea was to show the ability of the sample shaking/decantation extraction technique to work quantitatively even under unfavorable conditions. As the results of this experiment showed (Fig. 3), this proved to be the case. Recovery tests confirmed (Fig. 5) high absolute efficacy of the extraction. As expected, the number of washes required for extraction of 90 to $95 \%$ and over of nano-, micro-, and meiobenthic organisms was rather substantial (up to 10). Interestingly, this number corresponded well to the number of washes (8) found necessary to extract dislodged bacteria from sandy samples in similarly sized vials (Epstein \& Rossel 1995a).

The washing procedure could be easily shortened to 5 washes without compromising the extraction effi- 
cacy by a $50 \%$ increase in the WF/S ratio and by employing larger vials (Expt 2; Fig. 4). When samples were approximately $1.5 \mathrm{~cm}^{3}$, the WF/S ratio was 4.2 , and the vial size ( $25 \mathrm{~mm}$ o.d.) provided a large washing fluid-sediment surface interface area, the recovery was over $90 \%$ (nanobenthos) and over $95 \%$ (micro- and meiobenthos) after 5 washes. These estimates may be conservative as the results of the recovery experiments from azoic sediments (Fig. 5) suggest that the above numbers might be in fact just variations around $100 \%$.

It is of course impossible to test all the possible decantation protocols; other sample sizes, WF/S ratios, and vial diameters will result in different recovery efficacy. However, it appears likely that as long as samples are not larger, and WF/S ratios and vial diameters are not smaller, than those employed in Expt 2, the extraction (and overall enumeration) efficacy will be over $90 \%$ for nanobenthos and over $95 \%$ for larger microorganisms.

Once extracted, all target organisms could be efficiently cleaned from detritus by isopycnic centrifugation, which was first suggested by Starink et al. (1994) for nanoflagellate purification. Microorganisms (pigmented and colorless nano- and microflagellates, ciljates, nematodes, as well as occasionally seen harpacticoid copepods, turbellarians, tardigrades, and gastrotrichs), with a notable exception of diatoms, were retained within the liquid phase of the gradient (Fig. 2) whereas most of the detritus sedimented into a pellet.

The resulting mixture of purified organisms could be fluorescently stained [DAPI/FITC dual staining after Sherr \& Sherr (1983) and Sherr et al. (1993)] without gelling effect, and further concentrated on a filter without its clogging. None of the organisms examined, including the most fragile ciliates, such as Kentrophoros, Remanella, Loxophyllum, appeared to be damaged by either extraction, centrifugation, staining, or filter concentration. On typical filters, 100 to 200 nanobenthic organisms could be counted in 10 to 25 microscopic fields (400 to $600 \times$ magnification); the entire filter contained several thousand microflagellates, 100 to 300 ciliates, and 50 to 100 meiobenthic animals. The micro- and meiobenthic organisms could be easily counted by scanning either the entire filter or a portion thereof.

In conclusion, this protocol enables quick and efficient enumeration of marine nano-, micro-, and meiobenthos simultaneously from a single sandy sediment sample. It consists of the following steps:

(1) Sample is preserved with $1.5 \%$ glutaraldehyde in PFSW. To ca $1.5 \mathrm{~cm}^{3}$ sample in a $25 \mathrm{~mm}$ o.d. vial, $6 \mathrm{ml}$ of preservative (1 to $1.5 \%$ final concentration) were added in the present work.
(2) Sample is washed several times. Each time, 1.5\% glutaraldehyde in PFSW is added to the sample, sample is gently shaken for 10 to $15 \mathrm{~s}$, and supernatant is collected within a few seconds. All the supernatant fractions are later combined. In the case of the ca $1.5 \mathrm{~cm}^{3}$ samples in $25 \mathrm{~mm}$ o.d. vials, $6 \mathrm{ml}$ of washing fluid was used each time. Five washes were found to extract over $90 \%$ of nanobenthos and over $95 \%$ of micro- and meiobenthos; these estimates may be conservative.

(3) A subsample from combined supernatants is loaded on a gradiented Percoll solution. The latter is prepared after Starink et al. (1994) with minor modifications $150 \%$ Percoll in PFSW is centrifuged at $30000 \times g$ for $15 \mathrm{~min}$ ). In the present trials, $2 \mathrm{ml} \mathrm{sub-}$ samples were loaded over $4.5 \mathrm{ml}$ of Percoll gradient Percoll gradients were stable over time and could be stored in a refrigerator for at least $3 \mathrm{wk}$.

(4) Percoll gradient with the loaded sample is centrifuged for $15 \mathrm{~min}$ at $5000 \times g$ (Starink et al. 1994). The supernatant contains target organisms. It is collected by emptying the contents of the centrifuge vial into a disposable vial; the pellet at the bottom is normally firmly attached to the walls. The Percoll solution in supernatant does not gel in the presence of glutaraldehyde/fluorochromes. The extracted and cleaned organisms are stained with DAPI at $10 \mu \mathrm{g} \mathrm{ml}^{-1}$ final concentration for 10 to $15 \mathrm{~min}$. Stained organisms are concentrated on black polycarbonate membranes with appropriately sized pores ( $2 \mu \mathrm{m}$ pore-size filters were used throughout the study). After one 5 min washing in PFSW, the membranes are flooded with FITC solution prepared after Sherr et al. (1993) with the exception of the final FITC concentration used $\left(100 \mu \mathrm{g} \mathrm{ml}^{-1}\right)$, and, upon staining, washed again with PFSW.

(5) Filters are placed between drops of immersion oil and examined under appropriate epifluorescent illumination

Using this protocol, it was possible to enumerate nano-, micro-, and meiobenthic organisms with a rather high absolute efficiency. The protocol represents a novel way to enumerate nano-, micro-, and meiobenthos from sandy sediments. For nanobenthos, it represents the only approach quantitatively tested in sandy sediments. For microbenthos, it represents the first approach allowing for sample preservation. Perhaps most importantly, the protocol enables researchers to enumerate all the above organisms from a single sample on a single filter Compared to the 3 protocols run in parallel, the described technique reduces several-fold the time needed for nano-, micro-, and meiobenthos enumeration and makes quantification of these microorganisms as convenient as in water column research. In addition, this protocol is compatible with the recently modified grazing tech- 
nique (Epstein \& Rossel 1995b) which enables both enumeration of micrograzers and inspection of their digestive vacuoles/guts to be done on a single microscopic preparation.

Acknowledgements. The author expresses his appreciation of the support, help, and assistance he received throughout the duration of the project. Dr Sergei Sokol (Harvard University/Beth Israel Hospital) made his microscope facilities available. Jeffrey Rossel (Northeastern University) assisted at various stages of the work. Dr Nathan Riser (Northeastern University) corrected the English. The comments of 3 anonymous reviewers substantially improved and clarified the manuscript. Financial support came from NSF /Grant OCE9217250 to the author). This is contribution no. 212 of the Marine Science Center of Northeastern University, Nahant, MA, USA.

\section{LITERATURE CITED}

Alongi DM (1986) Quantitative estimates of benthic protozoa in tropical marine systems using silica gel: a comparison of methods. Estuar coast Shelf Sci 23:443-450

Alongi DM (1990) Abundances of benthic microfauna in relation to outwelling of mangrove detritus in a tropical coastal region. Mar Ecol Prog Ser 63:53-63

Alongi DM (1993) Extraction of protists in aquatic sediments via density gradient centrifugation. In: Kemp PF. Sherr BF. Sherr EB, Cole JJ (eds) Aquatic microbial ecology. Lewis Publ, Boca Raton, p 109-114

Bak RPM, Nieuwland G (1989) Seasonal fluctuations in benthic protozoan populations at different depths in marine sediments. Neth J Sea Res 24:37-44

Bak RPM, Nieuwland G (1993) Patterns in pelagic and benthic nanoflagellate densities in the coastal upwelling system along the Banc d'Arguin, Mauritania. Hydrobiologia 258:119-131

Bak RPM, van Duyl FC, Nieuwland G, Kop AJ (1991) Benthic heterotrophic nanoflagellates in North Sea field/meso$\cos m$ bottoms and their response to algal sedimentation. Ophelia 33:187-196

Berninger UG, Epstein SS (1995) Vertical distribution of benthic ciliates in response to the oxygen concentration in an intertidal North Sea sediment. Aquat microb Ecol 9: $229-236$

Caron D (1983) Technique for enumeration of heterotrophic and phototrophic nanoplankton, using epifluorescence microscopy, and comparison with other procedures. App] environ Microbiol 46:491-498

Epstein SS, Rossel J (1995a) Enumeration of sandy sediment bacteria: search for optimal protocol. Mar Ecol Prog Ser $117: 289-298$

Epstein SS, Rossel J (1995b) Methodology of in situ grazing experiments: evaluation of a new vital dye for preparation of fluorescently labeled bacteria. Mar Ecol Prog Ser 128: $143-150$

Epstein SS, Shiaris MP (1992) The rates of microbenthos- and meiobenthos bacterivory in a temperate muddy tidal-flat community. Appl environ Microbiol 58:2426-2431

Fenchel $\mathrm{T}$ (1975) The quantitative importance of the benthic microfauna of an arctic tundra pond. Hydrobiologia 46 : $445-464$

Responsible Subject Editor: F. Rassoulzadegan, Villefranchesur-Mer, France
Fenchel T (1987) Ecology of Protozoa. Springer-Verlag, Berlin Gasol JM (1993) Benthic flagellates and ciliates in fine freshwater sediments: calibration of a live counting procedure and estimation of their abundances. Microb Ecol 25:247-262 Giere O (1993) Meiobenthology. Springer-Verlag, New York Hondeveld BJM, Bak RPM, van Duyl FC (1992) Bacterivory by heterotrophic nanoflagellates in marine sediments measured by uptake of fluorescently labeled bacteria. Mar Ecol Prog Ser 89:63-71

Hondeveld BJM, Nieuwland G, van Duyl FC, Bak RPM (1994) Temporal and spatial variations in heterotrophic nanoflagellate abundance in North Sea sediments. Mar Ecol Prog Ser 109:235-243

Jonge VN, Bouwman LA (1974) A simple density separation technique for quantitative isolation of meiobenthos using the colloidal silica Ludox-TM. Mar Biol 42:143-148

Kemp PF (1990) The fate of benthic bacterial production. Rev aquat Sci 2:109-124

Lovejoy C, Vincent WF, Frenette JJ, Dodson JJ (1993) Microbial gradients in a turbid estuary - application of a new method for protozoan community analysis. Limnol Oceanogr 38:1295-1303

Mare MF (1942) A study of a marine benthic community with special reference to the microorganisms. J mar biol Ass UK 25:517-554

McIntyre AD, Warwick RM (1984) Meiofauna techniques. In: Holme NA, McIntyre AD (eds) Methods for the study of marine benthos. Blackwell, Oxford, p 217-244

Nichols JA (1979) A simple flotation technique for separating meiobenthic nematodes from fine-graded sediments. Trans Am Microsc Soc 98:127-130

Patterson DJ, Larsen J, Corliss JO (1989) The ecology of heterotrophic flagellates and ciliates living in marine sediments. Prog Protozool 3:185-277

Porter KG, Feig YC (1980) The use of DAPI for identifying and counting aquatic microflora. Limnol Oceanogr 25:943-948

Schallenberg M, Kalff J, Rasmussen JB (1989) Solutions to problems in enumerating sediment bacteria by direct counts. Appl environ Microbiol 55:1214-1219

Schwinghamer P (1981) Extraction of living meiofauna from marine sediments by centrifugation in a silica sol-sorbitol mixture. Can J Fish Aquat Sci 38:476-478

Sherr EB, Caron DA, Sherr BF (1993) Staining of heterotrophic protists for visualization via epifluorescence microscopy. In: Kemp PF, Sherr BF, Sherr EB, Cole JJ (eds) Aquatic microbial ecology. Lewis Publ, Boca Raton, $p$ 213-227

Sherr EB, Sherr BF (1983) Double-staining epifluorescence technique to assess frequency of dividing cells and bacterivory in natural populations of heterotrophic microprotozoa. Appl environ Microbiol 46:1388-1393

Sieburth J McN, Smetacek V, Lenz J (1978) Pelagic ecosystem structure: heterotrophic compartments of the plankton and their relationship to plankton size fractions. Limnol Oceanogr 23:1256-1263

Sokal RR, Rohlf FJ (1981) Biometry, 2nd edn. WH Freeman \& Co, New York

Starink M, Bär-Gilissen MJ, Bak RPM, Cappenberg TE (1994) Quantitative centrifugation to extract benthic protozoa from freshwater sediments. Appl environ Microbiol 60: $167-173$

Uhlig G (1964) Eine einfache Methode zur Extraktion der vagilen mesopsammalen Mikrofauna. Helgoländer wiss Meeresunters 11:178-185

Manuscript first received: September 13, 1995

Revised version accepted: October 23, 1995 